\title{
Glaucoma surgery preferences when the surgeon adopts the role of the patient
}

\author{
Ta C. Chang (1) ${ }^{1}$ Elizabeth A. Vanner $\mathbb{D}^{1} \cdot$ Richard K. Parrish II
}

Received: 22 November 2018 / Revised: 18 February 2019 / Accepted: 3 March 2019 / Published online: 1 May 2019

(c) The Royal College of Ophthalmologists 2019

\begin{abstract}
Background/Objectives To survey the members of the American Glaucoma Society (AGS) to determine which glaucoma procedures they would prefer to have performed on themselves.

Subjects/Methods We distributed an anonymous, electronic survey via the AGS listserv. The participants were asked to adopt the role of a patient with primary open angle glaucoma with progressive visual field loss in need of glaucoma surgery. Three preoperative intraocular pressure (IOP) levels were provided ( $>26 \mathrm{mmHg}, 21-26 \mathrm{mmHg}$, and $<21 \mathrm{mmHg}$ ), and the participants were asked to choose a glaucoma procedure they would prefer performed on themselves under each preoperative IOP levels from a list of fifteen procedures.

Results Out of 289 responses (representing 27.4\% of active and provisional AGS members), the most preferred procedures were ab interno trabeculotomy (20.3\%), Xen gel stent (18.6\%), iStent with two devices (14.3\%) and traditional trabeculectomy augmented with mitomycin C (14.1\%). $17.6 \%$ and $6.9 \%$ of participants preferred a trabeculectomy performed or a glaucoma drainage device (GDD) implanted on themselves, which is a lower than what would be offered to a hypothetical patient. Significant proportions of participants prefer non-bleb forming or conjunctiva-sparing procedures, even with low preoperative IOP levels. Older participants were more likely to prefer traditional trabeculectomy and having a single procedure across all levels of preoperative IOP.

Conclusions The majority of AGS participants in the survey would prefer micro-invasive glaucoma surgery over traditional trabeculectomy or a GDD performed on themselves as a primary glaucoma procedure, and most would prefer non-bleb forming and conjunctiva-sparing procedures.
\end{abstract}

\section{Introduction}

Glaucoma surgery can be viewed as a behavioral economics gamble, or prospect, with a certain probability of gain (i.e., long-term intraocular pressure [IOP] control, prevention of future loss of visual function, etc.) and loss (short- and long-term risks of complications, the time, effort and resources required to undergo surgery, etc.) [1]. The patient's preoperative status, such as visual acuity, IOP, disease severity, rate of glaucomatous progression and therapeutic burden, serves as a neutral reference point for the prospective outcomes. Ideally, a surgeon's decision to offer glaucoma surgery is rooted in

\footnotetext{
Ta C. Chang

t.chang@med.miami.edu

1 Bascom Palmer Eye Institute, Miami, FL, USA
}

the expected utility of the procedure. If the likelihood of benefits (gains) is greater than the risks (losses), then the procedure is offered as the best course of glaucoma management [2]. However, the perception of surgical risks may be different between a surgeon and a patient. Whereas the rational, expected utility model applies to a surgeon's decision to offer surgery, a patient's decision of whether to accept the surgeon's offer is likely guided by the prospect theory, which describes the behavioral economics for a decision under risk. In other words, if we assume the surgeon's decision as the utility-based "rational choice," a patient's decision may differ due to the cognitive and psychophysical determinants of making a risky decision, such as loss aversion and the desire to minimize short-term loss.

Prior studies have shown that physicians' recommendations are impacted by cognitive biases, and retinal specialists have been shown to select different age-related macular degeneration treatment for themselves than they 
would for a hypothetical patient [3, 4]. On the basis of this framework, we hypothesized that a glaucoma specialist's choice of surgery may differ based on context, e.g., when he or she adopts a patient's role (when the risks apply to themselves) versus a surgeon's role (when the risks apply to another person). We surveyed the members of the American Glaucoma Society (AGS) to determine which glaucoma procedures they would prefer to have performed on themselves if they were the patient, and compared the findings to that of a previous AGS survey on the surgical practice preferences regarding a hypothetical patient [5].

\section{Subjects and methods}

The study protocol was approved by the Institutional Review Board of the University of Miami Miller School of Medicine and the AGS Research Committee. We created an anonymous, electronic survey using an online survey tool (www.surveymonkey.com), and a link was distributed via e-mail to AGS members who subscribe to the AGS listserv on January 10, 2018. The survey was available for a period of 6 weeks and can be completed only once from each unique e-mail address.

The survey contained four questions. The participants were first asked to categorize themselves by age (30-40 years, 41-50 years, 51-60 years, 61-70 years, 71 or older), and were presented the following clinical scenario:

"You have primary open-angle glaucoma (without pseudoexfoliation or pigment dispersion) and progressive visual field loss with elevated intraocular pressure. You are taking all commercially available topical glaucoma medications and are adherent. You have had 360 degree SLT [selective laser trabeculoplasty] without adequate control. The visual acuity is 20/20 and you have no other signs or symptoms of ocular disease. You live in the United States and only have access to devices that have been approved by the FDA [Food Drug Administration]. What surgical option would you choose for yourself as your primary surgical procedure in the following preoperative situations?"

The participants were asked to choose one procedure from a list of fifteen surgical options (the procedures and the device manufacturers are listed in Table 1) under each of the three preoperative IOP levels: $>26 \mathrm{~mm} \mathrm{Hg}, 21-26 \mathrm{~mm} \mathrm{Hg}$, $<21 \mathrm{~mm} \mathrm{Hg}$.

Table 1 Surgical options listed in the survey for each of three preoperative scenarios and the frequencies chosen

\begin{tabular}{|c|c|c|c|c|c|}
\hline Surgical options & Bleb-forming & $\begin{array}{l}\text { Requires hardware } \\
\text { implant }\end{array}$ & Violates conjunctiva & Total frequency & Percent \\
\hline Trabeculotomy ab interno $\left(\mathrm{GATT}^{\mathrm{a}}\right.$ or $\left.\operatorname{Trab} 30^{\mathrm{b}}\right)$ & & & & 176 & 20.3 \\
\hline Xen gel stent ${ }^{b}$ & $\mathrm{x}$ & $\mathrm{x}$ & & 161 & 18.6 \\
\hline iStent (two devices) ${ }^{\mathrm{b}}$ & & $\mathrm{x}$ & & 124 & 14.3 \\
\hline $\begin{array}{l}\text { Trabeculectomy (traditional, augmented with } \\
\text { mitomycin C) }\end{array}$ & $\mathrm{x}$ & & $\mathrm{x}$ & 122 & 14.1 \\
\hline Canaloplasty, ab interno $\left(\mathrm{ABiC}^{\mathrm{a}}\right.$ or $\left.\mathrm{Visco} 360^{\mathrm{b}}\right)$ & & & & 61 & 7.1 \\
\hline Trabectome $^{b}$ & & & & 43 & 5.0 \\
\hline $\begin{array}{l}\text { Baerveldt glaucoma implant }{ }^{\mathrm{b}} \text { or other non- } \\
\text { valved device }\end{array}$ & $\mathrm{x}$ & $\mathrm{x}$ & $\mathrm{x}$ & 42 & 4.9 \\
\hline Cypass microstent $^{\mathrm{b}}$ & & $\mathrm{x}$ & & 38 & 4.4 \\
\hline $\begin{array}{l}\text { Trabeculectomy (ExPRESS }{ }^{\mathrm{b}} \text {, augmented with } \\
\text { mitomycin C) }\end{array}$ & $\mathrm{x}$ & $\mathrm{x}$ & $\mathrm{x}$ & 30 & 3.5 \\
\hline Ahmed glaucoma valve ${ }^{b}$ & $\mathrm{x}$ & $\mathrm{x}$ & $\mathrm{x}$ & 18 & 2.1 \\
\hline iStent (one device) ${ }^{\mathrm{b}}$ & & $\mathrm{x}$ & & 18 & 2.1 \\
\hline Canaloplasty, ab externo with suture stent & & & $\mathrm{x}$ & 12 & 1.4 \\
\hline Trabeculotomy, ab externo & & & $\mathrm{x}$ & 11 & 1.3 \\
\hline Deep sclerectomy (manual or laser-assisted) & & & $\mathrm{x}$ & 7 & 0.8 \\
\hline Canaloplasty, ab externo without suture stent & & & $\mathrm{x}$ & 2 & 0.2 \\
\hline Total & & & & 865 & 100 \\
\hline
\end{tabular}

${ }^{\mathrm{a}}$ GATT (gonioscopy-assisted transluminal trabeculotomy), ABiC (ab interno canaloplasty)

${ }^{\mathrm{b}}$ Ahmed glaucoma valve (New World Medical, Rancho Cucamonga, CA, USA), Baerveldt glaucoma implant (Johnson \& Johnson Vision, Jacksonville, FL, USA), Visco360 and Trab360 (Sight Sciences, Inc. Menlo Park, CA, USA), Cypass microstent (Alcon, Inc., Fort Worth, TX, USA), iStent (Glaukos, San Clemente, CA, USA), ExPRESS shunt (Alcon, Inc., Fort Worth, TX, USA), Trabectome (NeoMedix Corporation, Tustin, CA, USA), Xen gel stent (Allergan, Dublin, Ireland) 
Associations among categorical variables were assessed with the Pearson $X^{2}$ test for independence, Fisher's exact test for binary variables with small expected values, or the exact Pearson chi-square test for independence for nonbinary variables with small expected values. The Jonckheere-Terpstra Test was used to assess trends between two ordinal variables. The assessment of treatment choices among the three preoperative IOP levels $(>26 \mathrm{~mm} \mathrm{Hg}$, 21-26 $\mathrm{mm} \mathrm{Hg}$, and $<21 \mathrm{~mm} \mathrm{Hg}$ ) was done using the exact Pearson $X^{2}$ test and Fisher's exact test to compare binomial proportions, and the assessment of treatment choices within each preoperative IOP level was done using the $X^{2}$ test for specified proportions. All analyses were done using SAS version 9.4 (Cary, NC, USA).

\section{Results}

As of January 14, 2018, there were 786 active and 269 provisional AGS members (totaling 1055) (Nysather, personal communication). A total of 289 responses were received, which represented $27.4 \%$ of AGS members. Three participants did not provide the age range, one did not select a treatment with preoperative IOP $<21 \mathrm{mmHg}$, and one did not select a treatment for preoperative IOP $21-26 \mathrm{mmHg}$. Of the 289 participants, 141 (48.8\%) were 30-40 years of age (Table 2).

Given 289 participants and each were asked to choose the procedure they preferred under three different preoperative IOP levels, there were 867 possible choice outcomes (of which two were missing). Overall, ab interno trabeculotomy (gonioscopy-assisted transluminal trabeculotomy [GATT] or Trab360) was the most frequently chosen procedure $(176 / 865,20.3 \%)$, followed by the Xen gel stent $(161 / 865,18.6 \%)$, iStent with two devices $(124 / 865$, $14.3 \%$ ), and traditional trabeculectomy augmented with mitomycin C (MMC, 122/865, 14.1\%, Table 1).

Glaucoma drainage devices (GDD, Ahmed glaucoma valve, Baerveldt glaucoma implant or other non-valved devices) were chose 60 times out of 865 possible choice outcomes $(6.9 \%)$ across all preoperative IOP levels as the desired primary procedure. When chosen, glaucoma

Table 2 Age distribution of participants

\begin{tabular}{lrc}
\hline Age range & $N$ & Percent \\
\hline 30-40 years & 141 & 48.8 \\
41-50 years & 58 & 20.1 \\
51-60 years & 60 & 20.8 \\
61-70 years & 22 & 7.6 \\
71 years or older & 5 & 1.7 \\
Total & $286^{\mathrm{a}}$ & $99.0^{\mathrm{a}}$ \\
\hline
\end{tabular}

${ }^{\mathrm{a}}$ Three of 289 participants (1\%) did not provide the age range drainage devices were preferred when preoperative IOP is greater than $26 \mathrm{mmHg}(34 / 60,56.67 \%)$ compared to the other preoperative IOP groups $(p=0.0002)$. Traditional trabeculectomy or trabeculectomy with Ex-PRESS shunt were chosen 152 out of 865 possible choice outcomes $(17.6 \%)$ across all preoperative IOP levels. There were no significant differences in preference for among the preoperative IOP levels.

The top four most preferred procedures under each preoperative IOP levels are summarized in Table 3. In general, at lower preoperative IOP levels, more participants prefer non-bleb forming and/or conjunctiva-sparing procedures ( $p=0.0001$ and $p=0.0179$, respectively), while no clear preference exist for hardware-free procedures at any preoperative IOP levels $(p>0.2$, Table 3$)$. Traditional trabeculectomy was preferred by older participants (age 61 and older, Table 4).

Nearly two-fifths of the participants (113 of 285, 39.1\%) chose one single procedure for themselves across all levels of preoperative IOP, with a significant trend toward older age range having a stronger preference for a single across the board treatment (Table 5, $p=0.0124$ ).

Conversely, of those who did not choose the same treatment option for all levels of preoperative IOP, there is a significant tendency toward choosing non-bleb forming (36.2\%) and conjunctiva-sparing $(58.4 \%)$ procedures across all levels of preoperative IOP. There were no obvious preferences for choosing hardware-free procedures amongst this group.

\section{Discussion}

The process of deciding which glaucoma surgery to perform is complex, and involves a multitude of factors including the surgeon's training and experience, the procedure's perceived safety and efficacy, and the patient's preference. In this study, we asked AGS members to adopt the role of the patient, and to decide which procedure they would prefer to have performed on themselves in a hypothetical scenario. We made the following assumptions about the AGS survey participants - (1) they were consumers with intimate knowledge of the risks and benefits of glaucoma surgery, (2) they were patients with intimate knowledge of the natural history of glaucoma, (3) they knew how they would behave if the situation were actual and not merely hypothetical, and (4) they had no special reason to disguise their true preferences.

Overall, $17.6 \%$ and $6.9 \%$ of the AGS members who responded preferred to have a trabeculectomy (traditional or with the Ex-PRESS shunt, augmented with MMC) performed or a GDD implanted on themselves as a primary procedure. This is in contrast to the 2016 AGS member 


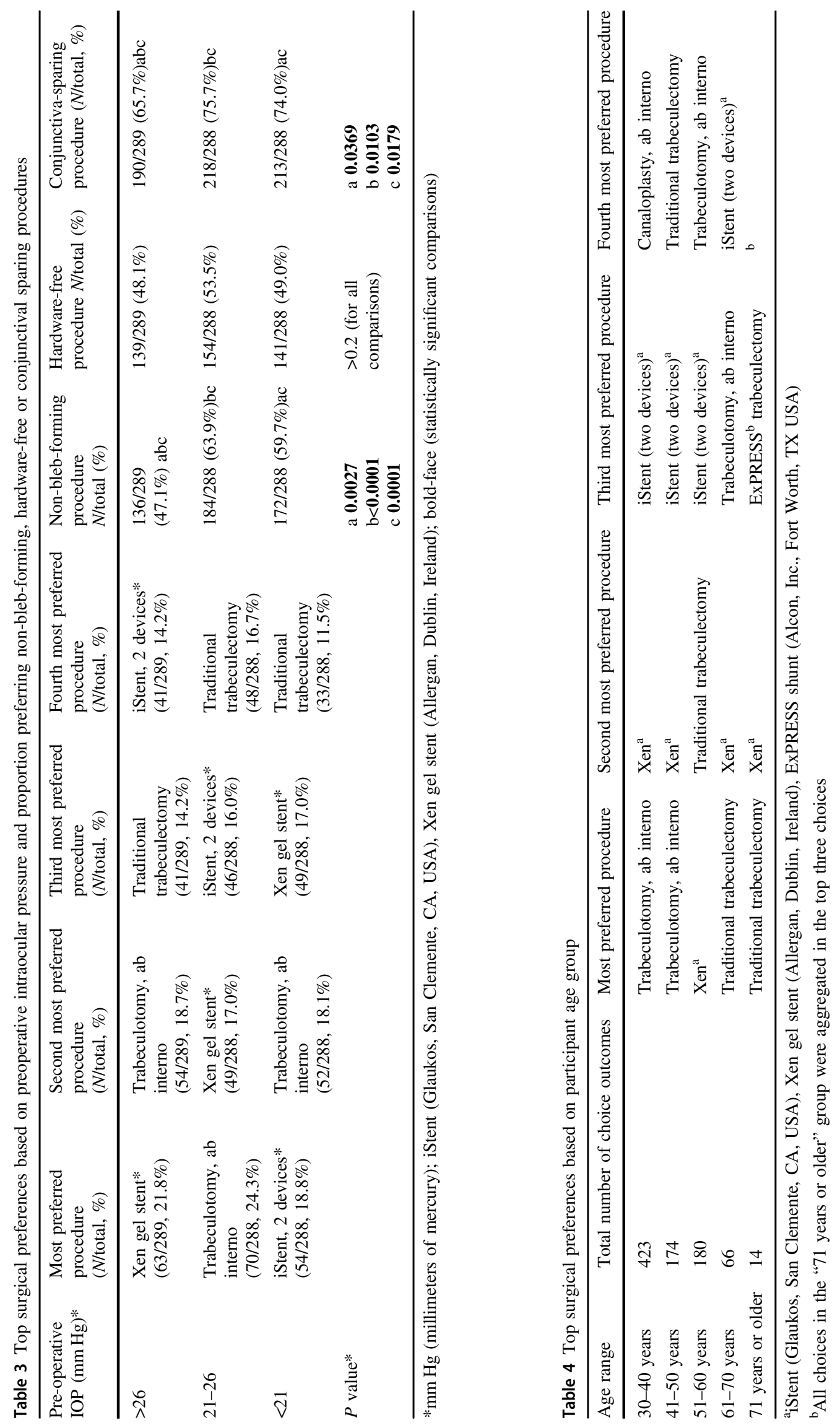


Table 5 Proportion of participants who would prefer the same procedure regardless of preoperative intraocular pressure versus those who prefer otherwise

\begin{tabular}{|c|c|c|c|c|c|c|}
\hline \multirow[t]{2}{*}{ Age range } & \multicolumn{2}{|c|}{$\begin{array}{l}\text { Same } \\
\text { procedure }\end{array}$} & \multicolumn{2}{|c|}{$\begin{array}{l}\text { Different } \\
\text { procedures }\end{array}$} & \multicolumn{2}{|c|}{ Total } \\
\hline & $N$ & Percent & $N$ & Percent & $N$ & Percent \\
\hline $30-40$ years & 44 & $31.2 \%^{\mathrm{a}}$ & 97 & $68.8 \%$ & 141 & $100 \%$ \\
\hline $41-50$ years & 29 & $50 \%^{\mathrm{a}}$ & 29 & $50 \%$ & 58 & $100 \%$ \\
\hline $51-60$ years & 29 & $48.3 \%^{\mathrm{a}}$ & 31 & $51.7 \%$ & 60 & $100 \%$ \\
\hline $61-70$ years & 8 & $36.4 \%^{\mathrm{a}}$ & 14 & $63.6 \%$ & 22 & $100 \%$ \\
\hline 71 years or older & 3 & $75 \%^{\mathrm{a}}$ & 1 & $25 \%$ & 4 & $100 \%$ \\
\hline Total & 113 & & 172 & & 285 & \\
\hline
\end{tabular}

${ }^{a}$ Trend analysis with Jonckheere-Terpstra Test demonstrated an overall increasing trend toward preferring the same procedure with older age, $p=0.0124$

survey of surgical practice preferences, in which approximately $59 \%$ and $23 \%$ of AGS members chose to offer a traditional trabeculectomy with MMC or a GDD to a hypothetical patient with primary open angle glaucoma (POAG) and no prior surgery [5]. In the traditional American pathway to becoming a glaucoma specialist, the glaucoma fellowship is typically completed around the age 30 . In the 2016 survey, 33\% of participants had finished fellowship training more than 20 years ago, whereas in the current survey, $30.1 \%$ of participants aged 51 years or more. This suggested comparable age distributions between the two surveys. Of all 15 surgical options listed, only Xen gel stent and Cypass microstent were approved following the 2016 survey, whereas the iStent was approved in 2012, and GATT was described in 2014 (both of which were chosen by a minority of participants in the 2016 survey) [6, 7]. This implied that any major paradigm shift toward favoring micro-invasive glaucoma surgery (MIGS) had occurred prior to either survey. Thus, the observed differences in surgical preferences were unlikely confounded by the introduction of new devices between surveys or a difference in age distribution. Since the efficacy (potential gain) of trabeculectomy or GDD remains the same when applied to the participants themselves and to hypothetical patients, this decreased preference of either procedure may be due an increase in the perceived risks (potential loss) when the participants adopted the patient role, which would support the notion that loss aversion can alter the perceived risk/ benefit ratio when an individual's perspective is shifted from that of a surgeon (expected utility theory) to that of a patient (prospect theory) [8].

In addition to loss aversion, the prospect theory also states that small changes near the neutral reference point have greater psychological impact than changes further from the reference point [8]. By adopting the patient role, the survey participants may have preferred procedures with low risks of short-term functional loss at the expense of a higher risk of long-term glaucomatous damage. The top two choices favored by the participants in this study, ab interno trabeculotomy and Xen gel stent, both have excellent short-term safety profiles. In a retrospective series, only $3 / 85(3.5 \%)$ patients who underwent GATT had complications more than 1 month after the surgery that was directly attributed to the procedure, none of which required reoperation [6]. Similarly, in a prospective series of Xen gel stent, 4/49 (9\%) of patients required reformation of the anterior chamber, while no other short-term complications were reported beyond the first postoperative month [9]. In contrast, 41 and $29 \%$ of patients who underwent a primary trabeculectomy or tube shunt implantation had complications beyond the first postoperative month, while $7 \%$ in the trabeculectomy group and $1 \%$ in the tube group required reoperation, experienced vision loss, or both [10]. In a large retrospective study, $28 \%$, 17 and $7 \%$ of patients required postoperative 5-fluorouracil injection, bleb needling, and resuturing for bleb leak or hypotony, respectively [11]. As the post-hoc analysis of the Advanced Glaucoma Intervention Study showed that the rate of glaucomatous progression is positively correlated with the IOP levels $[12,13]$, the participants' preference for safe but modestly effective glaucoma procedures on themselves over traditional filtering and shunting surgeries suggested a tendency of minimizing short-term loss in lieu of a higher (but more distant) risk of long-term loss, as described by the prospect theory [14].

Regardless of preoperative IOP levels, the majority of participants preferred conjunctiva-sparing procedures, while significant proportions also preferred non-bleb forming and hardware-free procedures. This is likely due to the perceived risks associated with bleb-forming and hardwarebased procedures. In primary tube shunts, 14/125 (11.2\%) and $17 / 125(13.6 \%)$ of patients experienced early and late postoperative complications, respectively, related to the presence of a bleb (shallow or flat anterior chamber, hypotony maculopathy, encapsulated bleb) or a hardware (plate erosion, tube retraction). Similarly, in primary trabeculectomy, 22/117 (18.8\%) and 16/117 (13.6\%) of patients experienced early and late postoperative complications attributed to the presence of a bleb [10]. At lower preoperative IOP levels, the participants were significantly more likely to choose non-bleb forming or conjunctivasparing procedures. One possible explanation is that the participants' aversion to bleb-forming or conjunctivaviolating procedures was sufficiently robust such that despite the glaucoma progressing at a low IOP level and their relative lack of efficacy, non-bleb forming and conjunctiva-sparing procedures were still preferred over bleb-forming and conjunctiva-violating alternatives. On the other hand, when faced with glaucomatous progression at a 
low IOP, the participants may have opted to try a relatively safe albeit modestly effective procedure first, in hopes of obviating an aggressive, bleb-forming surgery that may be needed in the near future.

There are several notable limitations to this study. First, whereas the 2016 AGS member survey on surgical preferences specified a hypothetical patient, our survey instructed the participants to adopt the patient role [3]. This approach can alter the impression of the hypothetical patient such that "a patient with POAG without prior surgery" in the 2016 survey may be imagined as elderly (fitting the profile of a "typical" POAG patient), whereas the young participant cohort in the current survey may view their own hypothetical disease closer to juvenile open-angle glaucoma rather than POAG. This altered perception may underlie the discrepancy of the proportion preferring trabeculectomy and GDD between the two surveys. Second, to be consistent with the 2016 survey format, no glaucoma severity was specified in the current survey. As the participants were adopting the patient role, most may assume that there is little or no functional vision loss despite the explicit description of "...progressive visual field loss" in the scenario text, as most participants probably do not have any functional vision loss in real life. This may exacerbate the loss aversion effect by providing a neutral reference point that does not closely approximate that of an average glaucoma patient in need of glaucoma surgery. Third, amongst the management choices, no surgery (and merely observation or augmenting with oral carbonic anhydrase inhibitor therapy) was not a choice. This may potentially be an important confounder because of "certainty effect" which describe that people tend to overweigh outcomes that are considered certain (the certainty to be free of any surgical complications) relative to outcomes which are merely probable (the risk of surgical complications, no matter how small) [8]. Fourth, we cannot discount the possibility of self-selection bias, such that a survey list with heavy emphasis on MIGS may attract more MIGS surgeons (as oppose to surgeons who have yet to adopt MIGS) to complete the survey. Fifth, our hypothetical scenario and surgical options was by no means comprehensive. Disease entities such as secondary open angle glaucoma or normal tension glaucoma were not presented, nor were surgical options such as the dual blade, goniotomy, cyclodestructive procedure or lens extraction alone. It is uncertain whether any of these choices would have affected the study outcome significantly. Sixth, our data may not be generalizable to non-American care delivery systems in which the medical and surgical glaucoma care may be rendered separately by different individuals. Lastly, while our response rate of $27.4 \%$ of AGS members may seem low, it is comparable to the historic control survey by Vinod et al. (23\% response rate) [5], and higher than a similar survey on retinal specialist practice preference differences when treating a hypothetical patient versus themselves $(20.1 \%)$ [15], and is considered an adequate sampling of the AGS members.

In summary, our data suggest that the glaucoma surgeon's surgical decisions may be impacted by cognitive biases rooted in the expected utility versus prospect theory models. The behavioral economics and cognitive biases of medical decision-making should be explored further in the future to optimize clinical outcomes and therapeutic designs.

\section{Summary}

\section{What was known before}

- Glaucoma surgeons offer surgery based on the expected utilities of surgery.

\section{What this study adds}

- When glaucoma surgeons adopt the roles of patients, their surgical preferences differed from what they would offer a patient, which suggests that the perception of surgical risk is dynamic depends on the prospective recipient of the surgery.

\section{Compliance with ethical standards}

Conflict of interest The authors declare that they have no conflict of interest.

Publisher's note: Springer Nature remains neutral with regard to jurisdictional claims in published maps and institutional affiliations.

\section{References}

1. Tversky A, Kahneman D. The framing of decisions and the psychology of choice. Science. 1981;211:453-8.

2. Chang TC, Ramulu PY, Hodapp EA. Clinical decisions in glaucoma. 2nd ed. Miami, FL/Charleston SC: Createspace Publishing; 2016.

3. Ubel PA, Angott AM, Zikmund-Fisher BJ. Physicians recommend different treatments for patients than they would choose for themselves. Arch Intern Med. 2011;171:630-4.

4. Jeng KW, Wilgucki J, Halperin S, Feuer WJ, Fine HF, Roth D, et al. Retina specialists treating age-related macular degeneration recommend different approaches for patients than they would choose for themselves. Retina. 2014;34:1796-801.

5. Vinod K, Gedde SJ, Feuer WJ, Panarelli JF, Chang TC, Chen PP, et al. Practice preferences for glaucoma surgery: a survey of the american glaucoma society. J Glaucoma. 2017;26:687-93.

6. Grover DS, Godfrey DG, Smith O, Feuer WJ, Montes de Oca I, Fellman RL. Gonioscopy-assisted transluminal trabeculotomy, ab 
interno trabeculotomy: technique report and preliminary results. Ophthalmology. 2014;121:855-61.

7. Saheb H, Ahmed II. Micro-invasive glaucoma surgery: current perspectives and future directions. Curr Opin Ophthalmol. 2012; 23:96-104.

8. Kahneman D, Tversky A. Prospective Theory: An Analysis of Decision under Risk. Econometrica . 1979;47:263-92.

9. Sheybani A, Dick HB, Ahmed II. Early clinical results of a novel $\mathrm{ab}$ interno gel stent for the surgical treatment of open-angle glaucoma. J Glaucoma. 2016;25:e691-6.

10. Gedde SJ, Feuer WJ, Shi W, Lim KS, Barton K, Goyal S, et al. Treatment outcomes in the primary tube versus trabeculectomy study after 1 year of follow-up. Ophthalmology. 2018;125:650-63.

11. Kirwan JF, Lockwood AJ, Shah P, Macleod A, Broadway DC, King AJ, et al. Trabeculectomy outcomes group audit study group. trabeculectomy in the 21 st century: a multicenter analysis. Ophthalmology . 2013;120:2532-9.

12. Palmberg P. Evidence-based target pressures: how to choose and achieve them. Int Ophthalmol Clin. 2004;44:1-14.

13. The AGIS Investigators. The Advanced Glaucoma Intervention Study (AGIS). 7. The relationship between control of intraocular pressure control and visual field deterioration. Am J Ophthalmol. 2000;130:429-40.

14. Tversky A, Kahneman D. Loss aversion in riskless choice: a reference-dependent model. Q J Econ. 1991;106:1039-61.

15. Wang MD, Jeng-Miller KW, Feng HL, Prenner JL, Fine HF, Shah SP. Retina specialists treating cystoid macular oedema secondary to retinal vein occlusion recommend different treatments for patients than they would choose for themselves. Br J Ophthalmol. 2016;100:1332-6. 